OPEN ACCESS

Edited by:

Tatsuo Kinashi,

Kansai Medical University, Japan

Reviewed by:

Yutaka Hata

Tokyo Medical and Dental University, Japan

Dae-Sik Lim

Korea Advanced Institute of Science and Technology (KAIST), South Korea

*Correspondence: Jinbo Cheng cheng_jinbo@126.com Zengqiang Yuan

zqyuan@bmi.ac.cn; zyuan620@yahoo.com

tThese authors share first authorship

Specialty section: This article was submitted to Multiple Sclerosis and

Neuroimmunology,

a section of the journal

Frontiers in Immunology

Received: 29 November 2019 Accepted: 04 February 2020

Published: 19 February 2020

Citation:

Cheng J, Wang S, Dong Y and Yuan Z (2020) The Role and Regulatory Mechanism of Hippo Signaling Components in the Neuronal System.

Front. Immunol. 11:281 doi: 10.3389/fimmu.2020.00281

\section{The Role and Regulatory Mechanism of Hippo Signaling Components in the Neuronal System}

\author{
Jinbo Cheng ${ }^{1,2 * t}$, Shukun Wang ${ }^{2 t}$, Yuan Dong ${ }^{3}$ and Zengqiang Yuan ${ }^{2 \star}$ \\ ${ }^{1}$ Center on Translational Neuroscience, College of Life and Environmental Science, Minzu University of China, Beijing, China, \\ ${ }^{2}$ The Brain Science Center, Beijing Institute of Basic Medical Sciences, Beijing, China, ${ }^{3}$ Department of Biochemistry, Medical \\ College, Qingdao University, Qingdao, China
}

The Hippo signaling pathway, an evolutionarily conserved protein kinase cascade, plays a critical role in controlling organ size, cancer development, and tissue regeneration. Recently, mounting evidence has suggested that Hippo signaling also has an important role in regulating immunity, including innate and adaptive immune activation. In the neuronal system, Our laboratory results, together with those from other studies, demonstrate that the Hippo signaling pathway is involved in neuroinflammation, neuronal cell differentiation, and neuronal death. In the present review, we summarize the recent findings pertaining to the function and regulatory mechanism of Hippo signaling components in the neuronal system, implicating the potential of Hippo signaling as a therapeutic target for the treatment of neuronal system diseases.

Keywords: Hippo signaling, oxidative stress, neuronal system, neuroinflammation, diseases

\section{INTRODUCTION}

The Hippo signaling pathway, originally identified in Drosophila, plays a critical role in regulating cell contact inhibition, proliferation, differentiation, and apoptosis. As such, this pathway is closely associated with the control of organ size, cancer development, and autoimmune diseases (1-6). Importantly, the canonical Hippo signaling pathway is a highly-conserved evolutionary pathway. As shown in Figure 1, the core components of this pathway in mammalian cells include mammalian Ste20-like kinases 1/2 (MST1/2 [orthologs of Hippo in Drosophila]) and their adaptor protein, Sav family WW domain containing protein 1 (SAV1 [orthologs of Salvador in Drosophila]). The phosphorylation of MST1/2 activates large tumor suppressor 1/2 [LATS 1/2 (orthologs of Warts in Drosophila)], a downstream protein, which in turn phosphorylates the downstream Yes-associated protein (YAP [ortholog of Yki in Drosophila]) or a transcriptional coactivator with PDZ-binding motif (TAZ); this results in the prevention of nuclear translocation by interacting with cytosolic protein 14-3-3. Meanwhile, unphosphorylated YAP or TAZ are relatively enriched in the nucleus and bind to their key transcriptional factors, the TEA domain transcription factor (TEAD) family [TEAD 1-4 (orthologs of Sd in Drosophila)]; thus regulating the expression of many genes that enhance cell proliferation, differentiation, and survival.

Although discovered in Drosophila, the Hippo signaling pathway in mammals also plays critical role in the control of organ size, cancer development, and tissue regeneration. Recently, accumulating evidence has suggested that Hippo signaling is important in regulating cancer immunity, and for innate and adaptive immunity (7-13). However, compared with the peripheral 
Drosophila

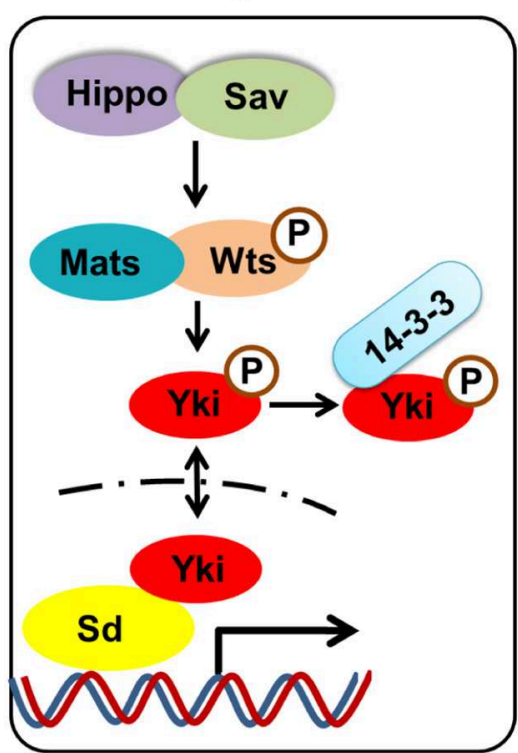

Mammal

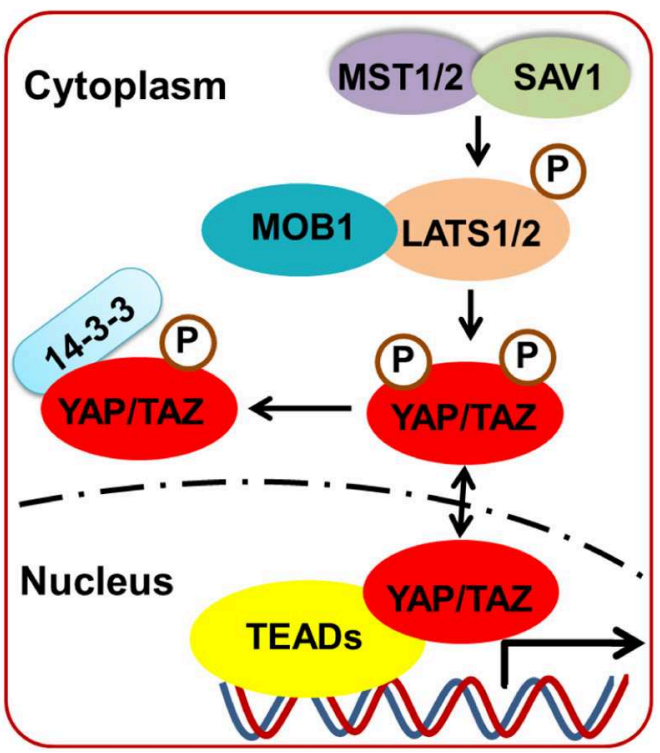

FIGURE 1 | The core components of the Hippo pathway in Drosophila and mammalian. The core components of Hippo signaling in mammalian cells include MST1/2 (orthologs of Hippo in Drosophila) and their adaptor protein SAV1 (orthologs of Salvador in Drosophila). The phosphorylated of MST1/2 activates LATS 1/2 (orthologs of Warts in Drosophila), which in turn phosphorylates the downstream YAP (ortholog of Yki in Drosophila) or TAZ, resulting in cytosolic retention by interacting with protein 14-3-3. Meanwhile, unphosphorylated YAP or TAZ which is relatively enriched in the nucleus, bind to their key transcriptional factors TEADs (orthologs of Sd in Drosophila), thus regulating the cell proliferation, differentiation, and survival.

immune system, the role and regulatory mechanism of this pathway in the neuronal system is less well-known. In the present review, we summarize the functions and regulation of the Hippo signaling pathway in the neuronal system, to update our understanding of this pathway and to raise awareness on its implications for drug development and the clinical treatment of disease.

\section{EXPRESSION OF THE HIPPO SIGNALING PATHWAY IN THE NEURONAL SYSTEM}

MST1 and MST2 are core components of the Hippo signaling pathway, that are highly expressed in several organs in mice. Double knockout (KO) of MST1 and MST2 results in early embryonic death (4); however, conventional deletion of MST1 or MST2 would only cause a failure of induction of tissue overgrowth or tumor development, thereby suggesting a functional redundancy in MST1 and MST2 (7). Consistent with this observation, organ overgrowth has been reported in many tissue-specific double KOs of MST1 and MST2, such as in the liver, intestine and heart $(3-5,14)$, suggesting that mammalian MST1 and MST2 are important in the regulation of development and growth.

Recently, mounting evidence has shown that the Hippo signaling pathway also plays a critical role in the neuronal system. Using an RNA-Seq transcriptome and splicing database of glia, neurons, and vascular cells of the cerebral cortex (15), we analyzed the expression levels of the core components of the Hippo signaling pathway. As shown in Table 1, MST1 and MST2 are highly expressed in most cell types in the brain, including astrocytes, neurons, oligodendrocyte progenitor cells (OPCs), newly formed oligodendrocytes, myelinating oligodendrocytes, microglia, and endothelial cells. The adaptor protein SAV1 also has a similar expression pattern, and downstream LATS1 and LATS2 are also highly expressed in all of these cell types. However, the co-transcription factor YAP is highly expressed only in astrocytes and endothelial cells, with low expression in neurons, OPCs, newly formed oligodendrocytes, myelinating oligodendrocytes, and microglia. Conversely, TAZ exhibits a different expression pattern compared with YAP, and it is highly expressed in all of these cell types, suggesting that YAP and TAZ may have different roles in the diverse cell types of the neuronal system. Different expression patterns have also been observed in TEAD 1, 2, 3, and 4. Specifically, TEAD1 is highly expressed in astrocytes and neurons, but relatively less expressed in OPCs and endothelial cells. TEAD2 is highly expressed in astrocytes and endothelial cells, while TEAD3 is expressed in astrocytes and neurons, with relatively lower expression levels in OPCs and microglia. TEAD4 on the other hand only has relatively high expression levels in endothelial cells. Hence, these diverse expression patterns of components of the Hippo signaling pathway suggest their diverse roles among the different cell types in the brain. 
TABLE 1 | The expressions of Hippo components in the brain.

\begin{tabular}{|c|c|c|c|c|c|c|c|}
\hline Gene name & \multicolumn{7}{|c|}{ Cell type in the brain } \\
\hline MST2(STK3) & $+^{\star *}$ & $+^{\star \star}$ & $t^{* \star}$ & $+^{\star *}$ & $+^{\star \star}$ & $+^{* *}$ & $t^{\star \star \star}$ \\
\hline SAV1 & $+^{\star \star \star}$ & $t^{\star \star}$ & $+^{\star \star \star}$ & $t^{\star \star}$ & $t^{\star \star}$ & $t^{\star \star \star}$ & $t^{\star \star \star}$ \\
\hline LATS1 & $+^{\star \star \star}$ & $++^{\star \star \star}$ & $+^{\star \star \star}$ & $+^{\star \star \star}$ & $+^{\star *}$ & $+^{\star *}$ & $+^{\star \star \star}$ \\
\hline YAP & $+^{\star \star \star}$ & $+^{*}$ & $+^{*}$ & + & + & + & $+t^{\star \star \star}$ \\
\hline$T Z A$ & $+^{\star \star \star}$ & $+^{\star \star \star}$ & $+^{\star \star \star}$ & $+^{\star \star \star}$ & $+^{\star \star \star}$ & $+^{\star \star \star}$ & $t^{\star \star \star}$ \\
\hline TEAD1 & $+^{\star * \star}$ & $+^{\star \star \star}$ & $+^{\star \star}$ & + & + & + & $+^{\star \star}$ \\
\hline TEAD2 & $+^{\star * \star}$ & + & + & - & - & + & $+^{\star \star \star}$ \\
\hline TEAD3 & $+^{\star \star \star}$ & $++^{\star \star \star}$ & $+^{\star \star}$ & + & + & $t^{\star \star}$ & + \\
\hline TEAD4 & + & + & + & + & + & + & $+^{\star \star}$ \\
\hline
\end{tabular}

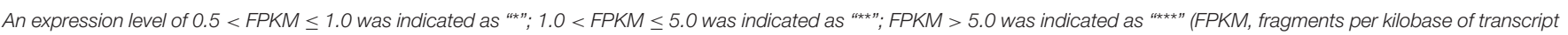
sequence per million mapped fragments).

\section{ROLE OF HIPPO SIGNALING IN NEURAL STEM CELLS}

In the vertebrate brain, neural stem cells (NSCs) are selfrenewing, multipotent cells that generate neurons and glial cells during embryonic development (16). It is noteworthy that some NSCs persisting in the subgranular and subventricular zones continue to produce neurons throughout life. Consequently, different states of NSCs exist and are tightly regulated in the brain. Usually, NSCs either undergo symmetrical or asymmetrical cell division into two daughter cells. In symmetrical cell division, both daughter cells are stem cells; however, in asymmetric division, NSCs produce differentiated daughter cells and stemness daughter cells (17). There are also some inactive state NSCs or quiescent NSCs, when proliferation is not required (18).

Recently, mounting evidence has shown that the Hippo pathway plays an important role in regulating NSC physiology. In neural progenitors, inactivation of LATS1/2 kinases (upstream inhibitors of YAP/TAZ) cause massive apoptosis through the induction of YAP/TAZ activation, and upregulating a series of genes associated with cell growth and proliferation (19). Additionally, overexpression of YAP/TAZ in the mouse embryonic brain induced cell localization in the ventricular zone by increasing stemness. Moreover, introduction of YAP/TAZ increased the frequency and size of neurospheres in a TEADdependent manner, as a TEAD binding-defective YAP mutant failed to induce this phenotype (20). The results from our study demonstrated that bone treatment with morphogenetic protein2 (BMP2) could inhibit the proliferation of embryonic NSCs; meanwhile, under the condition of YAP knockdown, BMP2 does not further reduce neurosphere formation, suggesting the presence of cross-talk between BMP2 signaling and the Hippo-YAP pathway. Mechanically, under BMP2 stimulation, Smad1/4 complex is transported into the nucleus, where it competes with TEAD1 for binding to YAP, resulting in the inhibition of its transcriptional activity. Furthermore, under the condition of cyclin D1 (ccnd1) knockdown, an important downstream target gene of YAP-TEAD signaling, BMP2 fails to show additional inhibitory effect on mouse NSC proliferation (21). YAP is also involved in neocortical astrocytic differentiation and proliferation during brain development in mice. Conditional KO of YAP, using Nestin-cre or GFAP-cre, decreases the number of neocortical astrocytes and impairs astrocytic proliferation through the BMP2-YAP-SMAD1 pathway (22). Furthermore, the loss of Hippo or Warts induces the growth and proliferation of NSCs in the Drosophila nervous system, suggesting that Hippo signaling also plays a critical role in maintaining NSC quiescence (23).

\section{ROLE AND MECHANISM OF HIPPO SIGNALING IN NEURONAL CELL DEATH}

It has been established that Hippo signaling is involved in the control of organ size and tumor development. Hippo inhibition results in higher activity of YAP and leads to tumorigenesis; however, its activation plays a role in neurodegeneration by mediating oxidative stress-induced neuronal death. Oxidative stress activates MST1 and then induces either YAP-dependent or YAP-independent cell proliferation and cell death $(24,25)$.

The mammalian fork-head transcription factors of the $\mathrm{O}$ class (FOXOs) are well-characterized substrates of MST1. By stimulating oxidative stress, we found that MST1 phosphorylates FOXO proteins, disrupts their interaction with protein 14-33 , and promotes FOXO nuclear translocation, thereby inducing cell death in neurons $(26,27)$. Apart from phosphorylation, we also found that methylation of $\mathrm{FOXO} 3$ was involved in neuronal cell death. Consequently, Methyltransferase Set9 methylates FOXO3 at lysine 270, leading to the inhibition of Bim expression and neuronal apoptosis (28). Moreover, we discovered that the upstream kinase $\mathrm{c}-\mathrm{ABL}$, a non-receptor 


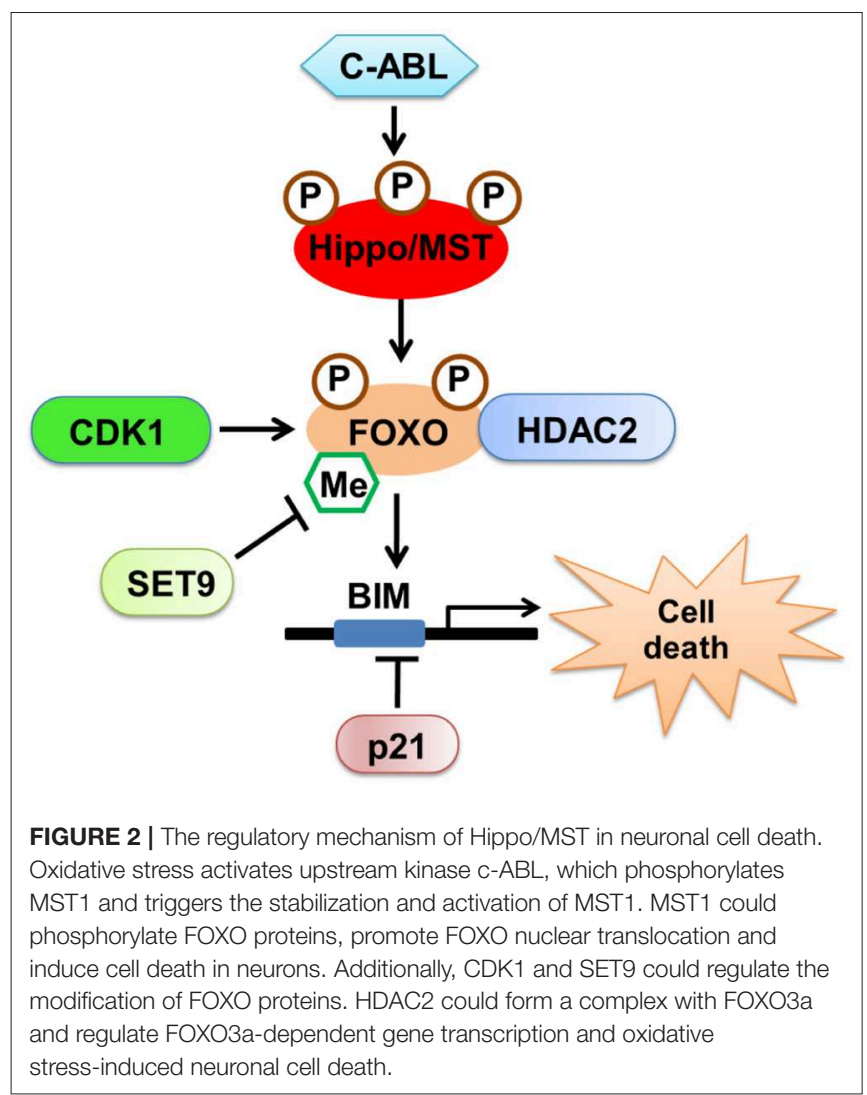

tyrosine kinase involved in the oxidative stress-induced neuronal cell death $(29,30)$, phosphorylates MST1 at Y433, which triggers the stabilization and activation of MST1, and increases the interaction between MST1 and FOXO3, thereby leading to neuronal cell death (31). Finally, we discovered that histone deacetylase 2 (HDAC2) could form a complex with FOXO3a and regulate FOXO3a-dependent gene transcription and oxidative stress-induced neuronal cell death, which describes a novel, epigenetic modification-dependent regulatory mechanism of FOXO3a-mediated selective gene transcription (32).

Interestingly, there is a functional interaction between Hippo-YAP signaling and FOXO1 in treatments that induce oxidative stress. YAP acts as a nuclear co-factor of FOXO1, which modulates the FOXO1-mediated antioxidant response. Activation of Hippo antagonizes YAP-FOXO1, leading to increased ischemia/reperfusion (I/R)-induced cell death through downregulation of catalase and MnSOD (33). These results revealed that MST1 could induce both YAP-dependent and YAP-independent gene transcription on oxidative stress, which both determine cell survival or death in the neuronal system (Figure 2).

\section{ROLE OF HIPPO SIGNALING IN NEURONAL SYSTEM DISEASES}

Accumulating evidence has shown that dysfunctions in Hippo signaling are involved in multiple neuronal system diseases. As shown in Table 2, in MST1 and MST2 KO mice, MST2-but not MST1-was shown to be a critical regulator of caspasemediated photoreceptor cell death in a mouse model of retinal detachment (RD). Mechanically, KO of MST2 decreases caspase-mediated photoreceptor cell death and proinflammatory cytokines, such as monocyte chemoattractant protein 1 and interleukin (IL)-6 during the early phase of RD (36). Moreover, MST1 has been reported to function as a key determinant of neurodegeneration in amyotrophic lateral sclerosis (ALS) (35). Furthermore, KO of MST1 delays disease onset and extends survival in mice expressing the human SOD1 G93A mutant. Mechanically, deficiency of MST1 also decreases the activation of p38 mitogen-activated protein kinase and caspases, and impairs autophagy in spinal cord motor neurons. Consistently, in Drosophila, Warts signaling is required for autophagic flux in neurons, and mutants of the Warts pathway cause progressive polyglutamine (PolyQ)-mediated neurodegeneration in the adult stage. Importantly, phosphorylated MST1-the active form of MST1-was reported to be significantly increased in the postmortem cortex of patients with Huntington's disease (HD). Meanwhile, YAP nuclear localization was decreased in both HD post-mortem cortex and neuronal stem cells derived from HD patients (24), suggesting that the activation of Hippo signaling may contribute to HD.

Additionally, MST1 was reported to be activated in a model of intracerebral hemorrhage established by injecting autologous blood into the right basal ganglia. Hence, genetic knockdown MST1 or chemical inhibition could effectively reduce the levels of p-LATS1 and p-YAP, and decrease neuronal cell death and inflammatory reactions, leading to a reduction in brain edema, blood-brain-barrier (BBB) damage, and neurobehavioral impairment (34). Furthermore, it has been reported that $I / R$ resulted in decreased levels of YAP and TAZ; hence, the intraperitoneal injection of the YAP agonist, dexamethasone, led to decreased BBB permeability, decreased cerebral edema, smaller brain infarct sizes, and improved neurological function, suggesting a neuroprotective effect of YAP on the I/R-induced damaged brain (37). Moreover, supplementation with melatonin could activate the YAP-Hippo pathway; thus enhancing mitochondrial fusion and ultimately reducing brain reperfusion stress. Mechanically, the YAPHippo pathway regulates melatonin-modified OPA1 expression, while blockade of the YAP-Hippo pathway results in neuronal cell death and mitochondrial damage (38). Additionally, the administration of biodegradable selenium (Se) nanoparticles led to the protection of axons in the hippocampus region and myelination of the hippocampal area after cerebral ischemic stroke. Mechanically, Se administration suppressed excessive inflammation and oxidative metabolism, and Hippo signaling was shown to be involved in this process (39).

\section{ROLE OF HIPPO SIGNALING IN NEUROINFLAMMATION}

Apart from the important role of Hippo signaling in controlling organ size and cancer development, its role in immunity 
TABLE 2 | The functions of Hippo components in the neuronal system diseases.

\begin{tabular}{|c|c|c|c|}
\hline Gene & Diseases & Function/change & References \\
\hline \multirow[t]{3}{*}{ Mst1 } & $\mathrm{ICH}$ & $\begin{array}{l}\text { Decrease neuronal cell death and inflammatory reaction, leading to the reduced brain edema, blood-brain } \\
\text { barrier damage, and neurobehavioral impairment }\end{array}$ & $(34)$ \\
\hline & ALS & $\begin{array}{l}\text { Decreased the activation of p38 mitogen-activated protein kinase and caspases, impaired the autophagy in } \\
\text { spinal cord motor neurons }\end{array}$ & (35) \\
\hline & HD & Phosphorylated MST1 increased in post-mortem HD cortex & $(24)$ \\
\hline Mst2 & $\mathrm{RD}$ & A critical regulator of caspase-mediated photoreceptor cell death & $(36)$ \\
\hline Wts & Aging & Causes progressive polyglutamine (PolyQ)-mediated neurodegeneration in the adult stage & $(24)$ \\
\hline \multirow[t]{2}{*}{ Yap } & HD & Decreased in the both HD post-mortem cortex and neuronal stem cells & $(24)$ \\
\hline & I/R & Decreased cerebral edema, smaller brain infarct sizes, and improved neurologic function & $(37)$ \\
\hline
\end{tabular}

ICH, intracerebral hemorrhage; ALS, amyotrophic lateral sclerosis; HD, Huntington's disease; RD, retinal detachment; I/R, ischemia/reperfusion.

activation has recently been extensively studied. The key component of the Hippo signaling pathway, MST1, is highly expressed in lymphoid tissues. MST1 KO mice also exhibit normal $\mathrm{T}$ cell development, but low numbers of mature naive $\mathrm{T}$ cells and relatively normal numbers of effector/memory $\mathrm{T}$ cells (7). In 2012, Abdollahpour et al. reported a homozygous premature termination mutation of MST1 with a novel clinical phenotype including $\mathrm{T}$ - and B-cell lymphopenia, intermittent neutropenia, and atrial septal defects; this suggest that MST1 deficiency is a novel human primary immunodeficiency syndrome. Moreover, enhanced loss of mitochondrial membrane potential and increased susceptibility to apoptosis was observed in MST1-deficient lymphocytes and neutrophils (8). In the same year, Nehme et al. reported a similar primary immunodeficiency phenotype associated with MST1 deficiency that was characterized by a progressive loss of naive $\mathrm{T}$ cells, recurrent bacterial and viral infections, and autoimmune manifestations (10). Subsequently, in 2015, Halacli et al. reported a novel STK4 mutation with clinical features including autoimmune cytopenias, viral skin and bacterial infections, mild onychomycosis, mild atopic, and seborrheic dermatitis, lymphopenia, and intermittent mild neutropenia (40); these features are similar with those of DOCK- 8 deficiency, a form of autosomal recessive (AR) hyperimmunoglobulin $\mathrm{E}$ syndrome. Hence, these results strongly indicate that MST1 plays a critical role in the immune system.

The diverse $\mathrm{KO}$ of major components of Hippo signaling models and some functional studies have also revealed that Hippo signaling plays an essential role in both innate and adaptive immunity. In innate immunity, the loss of Hippo or activation of Yki in fat bodies (the Drosophila immune organ) results in a decreased antimicrobial response and increase vulnerability to infection by Gram-positive bacteria. Mechanically, Gram-positive bacteria could activate Hippo-Yki signaling through Toll-Myd88 signaling, in which Yki directly regulates the transcriptional activity of Cactus, the Drosophila IкB homolog (11). However, an opposite role of Hippo signaling was shown in mammalian macrophages. Furthermore, MST1/2 deficient bone-marrow-derived macrophages exhibited higher toll-like receptor 4 -mediated nuclear factor (NF)- $\kappa \mathrm{B}$ activation, resulting in increased levels of some pro-inflammatory cytokines, such as IL-6, tumor necrosis factor-alpha, and IL-1 $\beta$ (41). Moreover, the downstream effector YAP was reported to negatively regulate antiviral immune response. Deficiency of YAP also resulted in enhanced innate immunity, and a decreased viral load, and morbidity in vivo (42). Additionally, YAP functions as a transcriptional coactivator of $\beta$-catenin in mesenchymal stem cell-mediated immune regulation. Deficiency of macrophage YAP or $\beta$-catenin increased XBP1-mediated NLRP3 expression, thus regulating macrophage polarization. In adaptive immunity, MST1 and MST2 have been demonstrated to also be important in $\mathrm{T}$ - and B-cell development, differentiation, and function (13, 43-45).

The functions of Hippo signaling in the neuronal system have recently been elucidated. We found that KO of MST1 in microglia protects from acute cerebral I/R-induced neuroinflammation and brain injury. Mechanically, in the acute cerebral I/R condition, MST1 directly phosphorylates IкB at residues S32 and S36, thus regulating the activation of NF- $\kappa \mathrm{B}$ signaling in microglia. Deficiency of MST1 in microglia significantly suppressed NF- $\kappa$ B signaling and microglial activation. Moreover, we found that Src kinase functions upstream of MST1-ІкB signaling, and that administration of the Src inhibitor AZD0530 exhibited a phenotype similar to MST1 deficiency in microglia (46). Consistent with this result, suppression of MST1 was also reported to reduce early brain injury after subarachnoid hemorrhage in mice by inhibiting NF-кB/MMP-9 signaling (47). These results suggest that MST1 positively regulates NF-кB signaling and that inhibition of MST1 plays a protective role in microglial activation-induced neuroinflammation. Moreover, YAP has been reported to be highly expressed in astrocytes, and YAP deletion induced the over-activation of astrocytes, along with microglial activation and $\mathrm{BBB}$ dysfunction in mice (48). Mechanically, KO of YAP in astrocytes increased the action of the JAK-STAT inflammatory pathway; thus, inducing reactive astrogliosis. Results from our laboratory demonstrated that YAP conditional $\mathrm{KO}(\mathrm{cKO})$ in the lens led to cataracts in mice (49). Mechanistically, YAP cKO reduced proliferation of epithelial cells, delayed fiber cell denucleation, and increased cellular senescence in the lens; the inflammation levels were also significantly altered in YAP cKO mice. Collectively, these results suggest that Hippo signaling is also important in 
neuroinflammation in the neuronal system, which may not be consistent with its functions in the peripheral immune system; thus, warranting further investigations.

\section{CONCLUDING REMARKS}

The Hippo signaling pathway is not only critical in controlling organ size, cancer development and tissue regeneration, but it is also important in regulating immunity, including the activation of the innate and adaptive immune systems. Recently, multiple studies have shown that Hippo signaling components paly critical role in the neuronal system, including the regulation neural stem cell proliferation and differentiation, oxidative stressinduced neuronal cell death, and in neuroinflammation; thus implicating a potential therapeutic target for the treatment of neuronal system diseases. However, the role and the

\section{REFERENCES}

1. Bai H, Zhang N, Xu Y, Chen Q, Khan M, Potter JJ, et al. Yes-associated protein regulates the hepatic response after bile duct ligation. Hepatology. (2012) 56:1097-107. doi: 10.1002/hep.25769

2. Camargo FD, Gokhale S, Johnnidis JB, Fu D, Bell GW, Jaenisch R, et al. YAP1 increases organ size and expands undifferentiated progenitor cells. Curr Biol. (2007) 17:2054-60. doi: 10.1016/j.cub.2007.10.039

3. Heallen T, Zhang M, Wang J, Bonilla-Claudio M, Klysik E, Johnson RL, et al. Hippo pathway inhibits Wnt signaling to restrain cardiomyocyte proliferation and heart size. Science. (2011) 332:458-61. doi: 10.1126/science.1199010

4. Zhou D, Conrad C, Xia F, Park JS, Payer B, Yin Y, et al. Mst1 and Mst2 maintain hepatocyte quiescence and suppress hepatocellular carcinoma development through inactivation of the Yap1 oncogene. Cancer Cell. (2009) 16:42538. doi: 10.1016/j.ccr.2009.09.026

5. Zhou D, Zhang Y, Wu H, Barry E, Yin Y, Lawrence E, et al. Mstl and Mst2 protein kinases restrain intestinal stem cell proliferation and colonic tumorigenesis by inhibition of Yes-associated protein (Yap) overabundance. Proc Natl Acad Sci USA. (2011) 108:E1312-20. doi: 10.1073/pnas.1110428108

6. Oh S, Lee D, Kim T, Kim TS, Oh HJ, Hwang CY, et al. Crucial role for Mst1 and Mst2 kinases in early embryonic development of the mouse. Mol Cell Biol. (2009) 29:6309-20. doi: 10.1128/MCB.00551-09

7. Zhou D, Medoff BD, Chen L, Li L, Zhang XF, Praskova M, et al. The Nore1B/Mst1 complex restrains antigen receptor-induced proliferation of naive $\mathrm{T}$ cells. Proc Natl Acad Sci USA. (2008) 105:20321-6. doi: 10.1073/pnas.0810773105

8. Abdollahpour H, Appaswamy G, Kotlarz D, Diestelhorst J, Beier R, Schaffer AA, et al. The phenotype of human STK4 deficiency. Blood. (2012) 119:34507. doi: 10.1182/blood-2011-09-378158

9. Crequer A, Picard C, Patin E, D’Amico A, Abhyankar A, Munzer M, et al. Inherited MST1 deficiency underlies susceptibility to EV-HPV infections. PLoS ONE. (2012) 7:e44010. doi: 10.1371/journal.pone.0044010

10. Nehme NT, Schmid JP, Debeurme F, Andre-Schmutz I, Lim A, Nitschke P, et al. MST1 mutations in autosomal recessive primary immunodeficiency characterized by defective naive T-cell survival. Blood. (2012) 119:345868. doi: 10.1182/blood-2011-09-378364

11. Liu B, Zheng Y, Yin F, Yu J, Silverman N, Pan D. Toll receptor-mediated Hippo signaling controls innate immunity in Drosophila. Cell. (2016) 164:40619. doi: 10.1016/j.cell.2015.12.029

12. Yang L, Li X, Qin X, Wang Q, Zhou K, Li H, et al. Deleted in azoospermiaassociated protein 2 regulates innate immunity by stimulating Hippo signaling in crab. J Biol Chem. (2019) 294:14704-16. doi: 10.1074/jbc.RA119.009559

13. Geng J, Yu S, Zhao H, Sun X, Li X, Wang P, et al. The transcriptional coactivator TAZ regulates reciprocal differentiation of TH17 cells and Treg cells. Nat Immunol. (2017) 18:800-12. doi: 10.1038/ni.3748 regulatory mechanism of Hippo signaling in the neuronal system still requires clarification, especially in different diseases or environmental conditions.

\section{AUTHOR CONTRIBUTIONS}

JC wrote the manuscript and prepared the figure. SW and YD review the manuscript. JC and ZY conceived the review topic and performed a comprehensive review of the literature.

\section{FUNDING}

This work was supported by grants from the National Nature Science Foundation of China (81870839; 81630026, and 31600946), the Beijing Nature Science Foundation (7161009) and the National Major Project of Support Program (AWS17J013).

14. Lee KP, Lee JH, Kim TS, Kim TH, Park HD, Byun JS, et al. The Hippo-Salvador pathway restrains hepatic oval cell proliferation, liver size, and liver tumorigenesis. Proc Natl Acad Sci USA. (2010) 107:824853. doi: 10.1073/pnas.0912203107

15. Zhang Y, Chen K, Sloan SA, Bennett ML, Scholze AR, O’Keeffe S, et al. An RNA-sequencing transcriptome and splicing database of glia, neurons, and vascular cells of the cerebral cortex. J Neurosci. (2014) 34:1192947. doi: 10.1523/JNEUROSCI.1860-14.2014

16. Beattie R, Hippenmeyer S. Mechanisms of radial glia progenitor cell lineage progression. Febs Lett. (2017) 591:3993-4008. doi: 10.1002/1873-3468

17. Belousov LV. Scott F. Gilbert-Developmental biology. (2010) Sinauer Associates, Inc., Sunderland, MA Ninth Edition. Russian J Dev Biol. (2011) 42:349. doi: 10.1134/S1062360411050043

18. Cheung TH, Rando TA. Molecular regulation of stem cell quiescence. Nat Rev Mol Cell Biol. (2013) 14:329-40. doi: 10.1038/nrm3591

19. Lavado A, Park JY, Pare J, Finkelstein D, Pan H, Xu B, et al. The Hippo pathway prevents YAP/TAZ-driven hypertranscription and controls neural progenitor number. Dev Cell. (2018) 47:576-91 e578. doi: 10.1016/j.devcel.2018.09.021

20. Han D, Byun S-H, Park S, Kim J, Kim I, Ha S, et al. YAP/TAZ enhance mammalian embryonic neural stem cell characteristics in a Tead-dependent manner. Biochem Biophys Res Commun. (2015) 458:1106. doi: 10.1016/j.bbrc.2015.01.077

21. Yao $M$, Wang $Y$, Zhang $\mathrm{P}$, Chen $\mathrm{H}$, Xu Z, Jiao J, et al. BMP2SMAD signaling represses the proliferation of embryonic neural stem cells through YAP. J Neurosci Off J Soc Neurosci. (2014) 34:1203948. doi: 10.1523/JNEUROSCI.0486-14.2014

22. Huang Z, Hu J, Pan J, Wang Y, Hu G, Zhou J, et al. YAP stabilizes SMAD1 and promotes BMP2-induced neocortical astrocytic differentiation. Development. (2016) 143:2398-409. doi: 10.1242/dev.130658

23. Ding R, Weynans K, Bossing T, Barros CS, Berger C. The Hippo signalling pathway maintains quiescence in Drosophila neural stem cells. Nat Commun. (2016) 7:10510. doi: 10.1038/ncomms 10510

24. Mueller KA, Glajch KE, Huizenga MN, Wilson RA, Granucci EJ, Dios AM, et al. Hippo signaling pathway dysregulation in human Huntington's disease brain and neuronal stem cells. Sci Rep. (2018) 8:11355. doi: 10.1038/s41598-018-29319-4

25. Yuan Z, Becker EB, Merlo P, Yamada T, DiBacco S, Konishi Y, et al. Activation of FOXO1 by Cdk1 in cycling cells and postmitotic neurons. Science. (2008) 319:1665-8. doi: 10.1126/science.1152337

26. Lehtinen MK, Yuan Z, Boag PR, Yang Y, Villen J, Becker EB, et al. A conserved MST-FOXO signaling pathway mediates oxidative-stress responses and extends life span. Cell. (2006) 125:987-1001. doi: 10.1016/j.cell.2006.03.046

27. Yuan Z, Lehtinen MK, Merlo P, Villen J, Gygi S, Bonni A. Regulation of neuronal cell death by MST1-FOXO1 signaling. J Biol Chem. (2009) 284:11285-92. doi: 10.1074/jbc.M900461200 
28. Xie Q, Hao Y, Tao L, Peng S, Rao C, Chen H, et al. Lysine methylation of FOXO3 regulates oxidative stress-induced neuronal cell death. EMBO Rep. (2012) 13:371-7. doi: 10.1038/embor.2012.25

29. Wu R, Chen H, Ma J, He Q, Huang Q, Liu Q, et al. c-Abl-p38alpha signaling plays an important role in MPTP-induced neuronal death. Cell Death Differ. (2016) 23:542-52. doi: 10.1038/cdd.2015.135

30. Zhou L, Zhang Q, Zhang P, Sun L, Peng C, Yuan Z, et al. cAbl-mediated Drp1 phosphorylation promotes oxidative stress-induced mitochondrial fragmentation and neuronal cell death. Cell Death Dis. (2017) 8:e3117. doi: 10.1038/cddis.2017.524

31. Xiao L, Chen D, Hu P, Wu J, Liu W, Zhao Y, et al. The c-Abl-MST1 signaling pathway mediates oxidative stress-induced neuronal cell death. J Neurosci. (2011) 31:9611-9. doi: 10.1523/JNEUROSCI.0035-11.2011

32. Peng S, Zhao S, Yan F, Cheng J, Huang L, Chen H, et al. HDAC2 selectively regulates FOXO3a-mediated gene transcription during oxidative stress-induced neuronal cell death. J Neurosci. (2015) 35:1250-9. doi: 10.1523/JNEUROSCI.2444-14.2015

33. Shao D, Zhai P, Del Re DP, Sciarretta S, Yabuta N, Nojima H, et al. A functional interaction between Hippo-YAP signalling and FoxO1 mediates the oxidative stress response. Nat Commun. (2014) 5:3315. doi: 10.1038/ncomms4315

34. Zhang P, Wang T, Zhang D, Zhang Z, Yuan S, Zhang J, et al. Exploration of MST1-mediated secondary brain injury induced by intracerebral hemorrhage in rats via Hippo signaling pathway. Transl Stroke Res. (2019) 10:72943. doi: 10.1007/s12975-019-00702-1

35. Lee JK, Shin JH, Hwang SG, Gwag BJ, McKee AC, Lee J, et al. MST1 functions as a key modulator of neurodegeneration in a mouse model of ALS. Proc Natl Acad Sci USA. (2013) 110:12066-71. doi: 10.1073/pnas.13008 94110

36. Matsumoto H, Murakami Y, Kataoka K, Lin H, Connor KM, Miller JW, et al. Mammalian STE20-like kinase 2, not kinase 1, mediates photoreceptor cell death during retinal detachment. Cell Death Dis. (2014) 5:e1269. doi: 10.1038/cddis.2014.218

37. Gong $\mathrm{P}$, Zhang Z, Zou C, Tian Q, Chen X, Hong $\mathrm{M}$, et al. Hippo/YAP signaling pathway mitigates blood-brain barrier disruption after cerebral ischemia/reperfusion injury. Behav Brain Res. (2019) 356:8-17. doi: 10.1016/j.bbr.2018.08.003

38. Wei N, Pu Y, Yang Z, Pan Y, Liu L. Therapeutic effects of melatonin on cerebral ischemia reperfusion injury: role of Yap-OPA1 signaling pathway and mitochondrial fusion. Biomed Pharmacother. (2019) 110:20312. doi: 10.1016/j.biopha.2018.11.060

39. Amani H, Habibey R, Shokri F, Hajmiresmail SJ, Akhavan O, Mashaghi A, et al. Selenium nanoparticles for targeted stroke therapy through modulation of inflammatory and metabolic signaling. Sci Rep. (2019) 9:6044. doi: 10.1038/s41598-019-42633-9
40. Halacli SO, Ayvaz DC, Sun-Tan C, Erman B, Uz E, Yilmaz DY, et al. STK4 (MST1) deficiency in two siblings with autoimmune cytopenias: a novel mutation. Clin Immunol. (2015) 161:316-23. doi: 10.1016/j.clim.2015.06.010

41. Geng J, Sun X, Wang P, Zhang S, Wang X, Wu H, et al. Kinases Mstl and Mst2 positively regulate phagocytic induction of reactive oxygen species and bactericidal activity. Nat Immunol. (2015) 16:1142-52. doi: 10.1038/ni.3268

42. Wang S, Xie F, Chu F, Zhang Z, Yang B, Dai T, et al. YAP antagonizes innate antiviral immunity and is targeted for lysosomal degradation through IKKvarepsilon-mediated phosphorylation. Nat Immunol. (2017) 18:73343. doi: $10.1038 /$ ni.3744

43. Thaventhiran JE, Hoffmann A, Magiera L, de la Roche M, Lingel H, BrunnerWeinzierl M, et al. Activation of the Hippo pathway by CTLA-4 regulates the expression of Blimp-1 in the CD8+ T cell. Proc Natl Acad Sci USA. (2012) 109:E2223-9. doi: 10.1073/pnas.1209115109

44. Onuora S. Immunology: Hippo signalling influences $\mathrm{T}$ cell fate. Nat Rev Rheumatol. (2017) 13:389. doi: 10.1038/nrrheum.2017.82

45. Hong L, Li X, Zhou D, Geng J, Chen L. Role of Hippo signaling in regulating immunity. Cell Mol Immunol. (2018) 15:1003-9. doi: 10.1038/s41423-018-0007-1

46. Zhao S, Yin J, Zhou L, Yan F, He Q, Huang L, et al. Hippo/MST1 signaling mediates microglial activation following acute cerebral ischemia-reperfusion injury. Brain Behav Immun. (2016) 55:236-48. doi: 10.1016/j.bbi.2015.12.016

47. Qu J, Zhao H, Li Q, Pan P, Ma K, Liu X, et al. MST1 suppression reduces early brain injury by inhibiting the NF-kappaB/MMP-9 pathway after subarachnoid hemorrhage in mice. Behav Neurol. (2018) 2018:6470957. doi: 10.1155/2018/6470957

48. Huang Z, Wang Y, Hu G, Zhou J, Mei L, Xiong WC. YAP is a critical inducer of SOCS3, preventing reactive astrogliosis. Cereb Cortex. (2016) 26:2299-310. doi: 10.1093/cercor/bhv292

49. He Q, Gao Y, Wang T, Zhou L, Zhou W, Yuan Z. Deficiency of yesassociated protein induces cataract in mice. Aging Dis. (2019) 10:293306. doi: 10.14336/AD.2018.0910

Conflict of Interest: The authors declare that the research was conducted in the absence of any commercial or financial relationships that could be construed as a potential conflict of interest.

Copyright (c) 2020 Cheng, Wang, Dong and Yuan. This is an open-access article distributed under the terms of the Creative Commons Attribution License (CC BY). The use, distribution or reproduction in other forums is permitted, provided the original author(s) and the copyright owner(s) are credited and that the original publication in this journal is cited, in accordance with accepted academic practice. No use, distribution or reproduction is permitted which does not comply with these terms. 